\title{
Attributable risk
}

National Cancer Institute ( $\mathrm{NCl})$

\section{Source}

National Cancer Institute (NCI). Attributable risk.

Proportion of a disease in exposed individuals that can be attributed to an exposure. In the context of genetic studies, the "exposure" is the frequency of a specific genetic variant. 